\title{
Physical ACTivity in Survivorship (PACTS): study protocol for a randomized controlled trial evaluating a goal-directed therapeutic exercise program in pediatric posterior fossa brain tumor survivors
}

\author{
Brooke E. Kohler ${ }^{1,2}$, Emmah Baque ${ }^{1,3}$, Carolina X. Sandler ${ }^{1,2,4}$, Denise S. K. Brookes ${ }^{1,2}$, Caroline O. Terranova ${ }^{1,2}$,
} Matthew Rixon ${ }^{5}$, Tim Hassall ${ }^{6}$ and Stewart G. Trost ${ }^{1,2^{*}}$ (i)

\begin{abstract}
Background: Posterior fossa brain tumors (PFBT) are the most common solid tumor in children. Recent increases in survival rates are encouraging; however, survivors may experience a plethora of disease- and treatment-related complications that can persist into adulthood. Therapeutic exercise interventions have been shown to improve quality of survivorship in other pediatric cancer diagnoses. There is also evidence that goal-directed interventions are effective at improving motor activities, function, and self-care in children with complex health conditions. Yet, there is currently no evidence on the efficacy of goal-directed therapeutic exercise in pediatric PFBT survivors. The Physical ACTivity in Survivorship (PACTS) study aims to investigate the effects of a novel goal-directed therapeutic exercise program on cardiorespiratory fitness and physical activity-related goal attainment in pediatric survivors of PFBT.
\end{abstract}

Method: PFBT survivors, aged five to 17 years, who underwent surgery at least 12 months earlier and completed radiation therapy and/or chemotherapy at least 6 months prior will be recruited from the Queensland Children's Hospital (Brisbane, Australia) (target $n=48$ ). Following baseline assessment, participants are randomized into either the intervention or usual care group. The intervention group will receive weekly individualized, goal-directed exercise therapy delivered face-to-face for 12 weeks, along with an accompanying home-based program (three sessions per week). Outcomes will be assessed at baseline, immediately post-intervention, and at 6- and 12-months postintervention. The primary outcomes are cardiorespiratory fitness $\left(\mathrm{Peak}_{\mathrm{VO}}\right)$ and physical activity-related goal attainment. Secondary outcomes are cardiorespiratory endurance, high-level mobility skills, functional muscle strength, habitual physical activity, gait, balance, quality of life, fatigue, participation, perceived movement skill competence and parameters of body composition.

(Continued on next page)

\footnotetext{
*Correspondence: s.trost@qut.edu.au

${ }^{1}$ Institute of Health and Biomedical Innovation at the Queensland Centre for

Children's Health Research, Queensland University of Technology (QUT),

Brisbane, Queensland, Australia

${ }^{2}$ School of Exercise and Nutrition Science, Queensland University of

Technology (QUT), Brisbane, Queensland, Australia

Full list of author information is available at the end of the article
} 
(Continued from previous page)

Discussion: PACTS is the first study to investigate the efficacy of goal-directed therapeutic exercise in children with PFBT and provide evidence needed to inform clinical practice recommendations for managing quality of survivorship in PFBT survivors.

Trial registration: ACTRN12619000841178.

Keywords: Oncology, Children, Physical activity, Cardiorespiratory fitness, Goal attainment, Quality of life

\section{Background}

Posterior fossa brain tumors (PFBT) account for $25 \%$ of all pediatric cancer diagnoses and are the most common solid tumor in children [1,2]. This cluster of diagnoses include medulloblastoma, ependymoma, cerebellar astrocytoma and brain stem glioma, with the former two showing increasing incidence rates over the last three decades [3, 4]. Advancements in medical management have led to significant improvements in survival, with five-year survival rates increasing by $5-10$, to $70 \%$ over the last 30 years (United States and Australia) [2, 4]. The improved survival rates of PFBTs presents an ongoing challenge of how to maximize quality of survivorship and effectively manage the short- and long-term complications acquired from the disease and treatment [3]. For the purpose of this study, a 'survivor' is regarded as any patient who has completed treatment with curative intent.

The anatomical location of PFBTs, with or without administration of cranial radiation therapy, negatively impact the cerebellum and adjacent areas [5]. Consequently, pediatric survivors of PFBTs may experience several late effects following treatment, including motor, neurological, cognitive, and psychosocial complications [5-9]. Compared to typically-developing peers, childhood PFBT survivors are less able to participate in physical activity, maintain schoolwork, engage in conversations with family and peers, and participate in other age-appropriate activities such as learning to drive or household chores [10, 11]. Moreover, PFBT survivors report significantly poorer quality of life compared to healthy controls and other types of cancers [12,13]. Fatigue is frequently reported in childhood cancer survivors, with nearly half experiencing persistent fatigue symptoms months to years after completion of treatment [14]. Fatigue may impact children's meaningful engagement in school, recreation and home activities, through poor concentration, decreased attention, memory loss and activity intolerance $[14,15]$. Mechanisms that underpin the activity limitations and participation restrictions associated with a PFBT and its treatment persist through many years of survivorship and increase an individual's risk for disabling chronic health conditions. In adulthood, PFBT survivors exhibit higher rates of cardiovascular disease, obesity, diabetes and osteoporosis than the general population [16-19].
Pediatric PFBT survivors exhibit poor cardiorespiratory fitness, performing well below their age-matched peers and equivalent to children with congenital heart disease $[6,20]$. Following treatment, PFBT survivors also demonstrate significant deficits in lower limb strength, balance, and motor proficiency $[6,8,21,22]$. These physical impairments have been shown to persist into adulthood, with young adult survivors of childhood brain tumors demonstrating similar muscle strength to persons aged over 60 years [7, 23]. Survivors of childhood cancer engage in less physical activity than their typically-developing age-matched peers; and brain tumor survivors are less likely to meet physical activity guidelines than children with other cancer diagnoses [24-26]. Lifestyle factors such as physical inactivity are associated with increased risk of chronic disease, and regular participation in physical activity has been shown to be both preventative and therapeutic [27].

Intervention studies conducted in childhood cancer survivors indicate that therapeutic exercise is safe and feasible; however, studies conducted to date have focused predominantly on children with blood cancers (e.g., acute lymphoblastic leukemia) [28]. To our knowledge, there are no published randomized controlled trials evaluating the efficacy of therapeutic exercise in PFBT survivors. Results from small uncontrolled trials suggest that therapeutic exercise can positively impact a range of functional outcomes, including cardiorespiratory fitness, strength and bilateral coordination [29-32]. Furthermore, therapeutic exercise is associated with positive changes in brain architecture, which has been linked to improvements in motor planning, cognitive function and behavioral outcomes [33, 34]. Exercise interventions are also reported to enhance quality of life and reduce fatigue in childhood cancer survivors, but this has not been rigorously examined in PFBT survivors $[28,35,36]$.

Goal-directed therapy aims to promote functional performance and independence in everyday activities, where goals are set by the patients and their families [37, 38]. An individualized, patient-centered approach not only promotes attention and motivation in therapy, but also provides a platform for delivering fully personalized therapeutic exercise programs for patients with significant impairments, activity limitations, and participation 
restrictions [39]. Goal-directed therapy aligns with the principles of the International Classification of Functioning, Disability and Health (ICF) framework, with particular focus on the activity and participation domains [40]. There is evidence supporting the effectiveness of familycentered, goal-directed interventions to improve motor performance, habitual physical activity, and quality of life in other pediatric patient groups, including cerebral palsy, muscular dystrophy, and intellectual disability [37, $39,41]$. No studies, however, have evaluated the efficacy of a goal-directed exercise intervention in any pediatric brain tumor survivor cohort.

\section{Aims and hypotheses}

The Physical ACTivity in Survivorship (PACTS) study aims to evaluate the effects of a 12-week goal-directed therapeutic exercise program on cardiorespiratory fitness and physical activity-related goal attainment in PFBT survivors. We hypothesize that, compared with usual care, children who receive the intervention will achieve significantly greater gains in cardiorespiratory fitness and goal attainment, immediately post-intervention, with improvements maintained at 6- and 12-month follow-ups.

Secondary aims are to evaluate the effects of the exercise program on cardiorespiratory endurance, high-level mobility skills, functional muscle strength, habitual physical activity, gait, balance, quality of life, fatigue, participation, perceived movement skill competence and parameters of body composition. We hypothesize that, compared with usual care, children who receive the intervention will achieve significantly greater gains in these secondary outcomes immediately post-intervention, with improvements maintained at 6-and 12-months follow-ups.

\section{Methods}

\section{Study design}

This study is a single-center randomized controlled trial. Participants will be randomized into the intervention arm $(n=24)$ or usual care $(n=24)$. The study will be conducted in accordance with the Standard Protocol Items: Recommendations for Interventional Trials (SPIR IT) guidelines [42]. See SPIRIT Checklist in Additional file 1. Assessments for all participants will be conducted at baseline (T1), immediately post-intervention (12-weeks post baseline) (T2), and at 6-month (T3) and 12-month (T4) follow-up. Each assessment involves: 2 days of testing at a clinic visit, assessor-administered or selfadministered (age dependent) child questionnaire, selfadministered parent questionnaire, and monitoring of physical activity by accelerometer for 7 days. An overview of the study design, the schedule for enrollment and study assessments is shown in Table 1.

\section{Eligibility criteria}

To be eligible participants must: be aged between five and 17 years; have had a diagnosis of a PFBT; undergone surgery for a PFBT a minimum of 12 months earlier, with no maximum time since surgery providing age eligibility is met; have completed radiation and/or chemotherapy in the previous 6 months; have no evidence of progressive disease; and, are deemed medically able to complete an exercise program, as determined by the treating oncologist. Participants will be excluded if: they have insufficient literacy skills to complete study assessments; or they are unable to travel to the research facility to complete study assessments or exercise intervention sessions.

\section{Recruitment}

Potentially eligible participants will be identified through a patient database at the Pediatric Oncology Unit at Queensland Children's Hospital, Brisbane, Australia. Potential participants may also be identified at weekly Pediatric Oncology multi-disciplinary meetings. Identified participants will be contacted by a Neuro-oncology Clinical Nurse via telephone, email, mail, or in person during their out-patient appointment, where they will be provided with a study information packet and eligibility screening will be completed. Following confirmation of eligibility and informed consent, a member of the research team will schedule the baseline assessment.

\section{Randomization, allocation, and blinding}

The treatment allocation schedule will be generated and supervised by an independent statistician and concealed in Research Electronic Data Capture (REDCap). Using the randomization module in REDCap, participants will be randomized into the intervention or usual care group, stratified by treatment type (surgery only; surgery and radiation therapy; surgery, radiation therapy and chemotherapy). Within an exercise intervention, it is not possible to blind either therapist or participant; however, therapists will not take part in post-intervention outcome assessments. Trained assessors blinded to group allocation will collect outcome data.

\section{Therapeutic exercise intervention}

Participants randomized to the intervention group will receive weekly individualised, goal-directed exercise therapy delivered face-to-face for 12 weeks, along with an accompanying home-based program. Each face-to-face session will be delivered by a qualified physiotherapist or accredited exercise physiologist (referred to hereafter as therapist), for a 30-60-min duration. The intervention will adhere to accepted exercise prescription guidelines for children and adolescents, comprising physical exercise training (cardiorespiratory and strength-promoting activities) and 
Table 1 Schedule of enrollment, intervention, and assessment

\begin{tabular}{|c|c|c|c|c|c|c|}
\hline \multirow[b]{4}{*}{ TIMEPOINT } & \multirow{4}{*}{$\begin{array}{c}\text { Screening } \\
\text { TO }\end{array}$} & \multicolumn{5}{|c|}{ STUDY PERIOD } \\
\hline & & \multicolumn{2}{|c|}{ Baseline and Allocation } & \multicolumn{3}{|c|}{ Post-allocation } \\
\hline & & $T 1$ & \multirow[b]{2}{*}{ Allocation } & $T 2$ & $T 3$ & $T 4$ \\
\hline & & Baseline & & $\begin{array}{c}\text { End of } \\
\text { intervention } \\
12 \text { weeks }\end{array}$ & $\begin{array}{l}\text { Follow-up } \\
6 \text { months }\end{array}$ & $\begin{array}{l}\text { Follow-up } \\
12 \text { months }\end{array}$ \\
\hline \multicolumn{7}{|l|}{ ENROLLMENT: } \\
\hline Inclusion criteria & $x$ & & & & & \\
\hline Eligibility screen & $x$ & & & & & \\
\hline Informed consent & $\mathrm{x}$ & & & & & \\
\hline Allocation & & & $x$ & & & \\
\hline \multicolumn{7}{|l|}{ GROUPS: } \\
\hline \multicolumn{7}{|l|}{ Exercise intervention } \\
\hline \multicolumn{7}{|l|}{ Usual care control } \\
\hline \multicolumn{7}{|l|}{ ASSESSMENTS: } \\
\hline $\begin{array}{l}\text { Demographic and } \\
\text { medical information }\end{array}$ & & $\mathrm{x}$ & & & & \\
\hline Primary outcomes & & $x$ & & $x$ & $x$ & $x$ \\
\hline Secondary outcomes & & $x$ & & $x$ & $x$ & $x$ \\
\hline Program satisfaction & & & & $x$ & & \\
\hline \multicolumn{7}{|l|}{$\begin{array}{r}\text { Adherence to exercise } \\
\text { intervention }\end{array}$} \\
\hline Adverse events & & & $\leftarrow$ & & & \\
\hline
\end{tabular}

Primary outcomes: cardiorespiratory fitness and physical activity-related goal attainment

Secondary outcomes: cardiorespiratory endurance, high-level mobility skills, functional muscle strength, habitual physical activity, gait, balance, quality of life,

fatigue, participation, perceived movement skill competence and parameters of body composition

goal-directed task specific practice [43, 44]. At least half of the face-to-face session will be dedicated to cardiorespiratory-focused activities, with the remaining half dedicated to goal-directed activities. Goals selected will be physical activity- related and may include community recreation, sports, or leisure goals. The structure of the therapeutic exercise program, including the type, intensity and duration, will be individualized according to each participant's age, goals, interests, functional capacity, presence of treatment-related side-effects and co-morbidities.

To supplement the face-to-face sessions, participants will complete a parent-led, home-based exercise program three times per week, up to $30 \mathrm{~min}$ of duration each time. The home-based program will replicate the essential elements of the supervised sessions in relation to activity type, intensity, and duration, and will also reflect the resources available at home. Prior to commencing face-to-face sessions, the therapist will complete a home visit to determine what space, equipment, and resources are available to complete the home-based exercise program safely and effectively. Parents will be given a detailed manual (including pictures and descriptions) for delivering the home-based component and will be encouraged to participate in the activities with their child. Peers and siblings may also be included to promote adherence to the home-based program. The home-based program will be monitored via heart rate monitors. The therapist will complete a follow-up home visit, between week four and week eight of the intervention, to provide an opportunity for participants and parents to ask questions about the home-based program, resolve any difficulties completing the program, and review the current activities. 


\section{Program fidelity}

Due to the highly heterogenic nature of a goaldirected intervention, several strategies will be used to monitor compliance with the exercise program. First, the type, intensity and duration of each activity will be documented by the therapist using a standard form. The forms will prompt therapists to reflect on whether their session delivered the required dose of cardiorespiratory and goal-directed activities. Second, where possible, all sessions will be delivered by the same therapist to ensure consistency between sessions. Third, when consent is provided by parents or their guardian, intervention sessions will be video recorded and assessed by another member of the research team, with significant experience in pediatric exercise therapy. At the conclusion of the exercise intervention, parents will be asked to complete a questionnaire assessing their overall satisfaction with the program and whether the program met their expectations and needs.

\section{Usual care}

Participants randomized to the control group will receive care as usual. Usual care is highly variable for children who are PFBT survivors; however, it is anticipated that most participants will not receive direct therapy services for the duration of the study. Usual care may include any therapy (e.g., physiotherapy, occupational therapy, speech and language therapy) and visits to their treating medical practitioner.

\section{Participant characteristics}

Upon meeting eligibility criteria and providing written consent, participants will complete a demographic and medical information questionnaire, provided to the parent up to 2 weeks prior to their scheduled baseline assessment. The following medical information will also be extracted from patient medical records by trained staff: definitive date of diagnosis, tumor type and grade, intraventricular shunt placement, extent of surgery, site and dose of radiation therapy, agent and cumulative dose of chemotherapy, hormone therapy, dose and frequency of Botulinum Toxin A (BoNTA), and physical impairment (e.g., ataxic gait, hemiplegia).

At each assessment, the following anthropometric measures will be taken: height (Seca 264 digital stationary stadiometer, Seca, Germany), weight (Seca 703, column scales, Seca, Germany), and waist circumference measured using a stretch resistant tape. Information about each participant's usual care during the study period will be collected through a semi-structured parent interview.

\section{Primary outcomes}

Cardiorespiratory fitness

A continuous ramped exercise test for determination of maximal aerobic capacity will be conducted on an electronically braked pediatric cycle ergometer (Ergoselect 150, Ergoline, Bitz, Germany). Following a 2-min warmup at an output of 10 watts, power output will increase at a rate of $10 \mathrm{~W}$ per minute and cycling cadence will be maintained at $75 \mathrm{rpm}$ (rpm) [45]. The test will continue until pedal cadence drops below $60 \mathrm{rpm}$ for 5 consecutive seconds, at which point power output will be reduced to $10 \mathrm{~W}$ for a 5-min recovery. Peak oxygen consumption (Peak $V \mathrm{O}_{2}$ ) will be determined using the methods described by Day et al. [46]. Breath-by-breath changes in gas exchange and ventilation will be measured using the MetaMax-3B portable metabolic analyzer (Cortex, Leipzig, Germany). Oxygen consumption $\left(V \mathrm{O}_{2}\right)$ will be averaged over five second time windows. This measure has been shown to be reliable among children $(\mathrm{ICC}=0.96)$ [47].

\section{Goal attainment}

The Canadian Occupational Performance Measure (COPM) is a semi-structured interview used to identify participant issues of personal importance in the domains of self-care, productivity and leisure [48]. Only goals pertaining to active recreation will be considered for this study. Children and parents will be asked to identify activities the child has difficulty performing, and to rate each activity on a scale of importance, rated from 1 (lowest) and 10 (highest). Up to five priority goals will be identified using the importance rating, and these are then rated on a scale of performance and satisfaction, using a similar 10-point scale [49]. The COPM will be administered during baseline assessment. The goals set by children allocated to the intervention group will be used to guide activity selection for the face-to-face intervention sessions and home-based program. Ratings from the parent for children under 8 years of age, and ratings from children over 8 years, in collaboration with their parent, will be reported $[50,51]$. The COPM test-retest reliability is high (ICC $0.76-0.89$ ) and is responsive to change in pediatric clinical trials $[40,52,53]$. A change of two points or greater has been reported as being clinically significant [48].

\section{Secondary outcomes \\ Cardiorespiratory endurance}

The Modified Shuttle Test requires participants to walk/ run back and forth along a $10-\mathrm{m}$ course in time with a sound signal recording [54]. Speed for the first minute is set to $1.8 \mathrm{~km} /$ hour and increased by $0.61 \mathrm{~km} /$ hour every minute thereafter. The test is terminated when participants can no longer follow the pace set by the recording 
and the last lap successfully completed is recorded [55]. Heart rate and perceived effort using the OMNI scale will be recorded just prior to the test and immediately following test termination [56]. The Modified Shuttle Test has been used in pediatric patients with cystic fibrosis and has been shown to be a reproducible and valid measure of cardiorespiratory endurance $[55,57]$.

\section{High-level mobility skills}

High-Level Mobility Assessment Tool (HiMAT) is a 13item tool assessing the performance of high-level mobility skills including walking, running, skipping, hopping, stairs, and bounding, among people who have sustained a brain injury [58]. Raw scores for all items will be recorded and scored on a scale between zero and four, where a score of zero represents the inability to perform an item, and a score of one to four represents increasing levels of ability. An additional five points for each stair item, will be awarded to the participant if they can ascend and/or descend the stairs using a reciprocal lower limb pattern and without the assistance of a rail. The HiMAT has demonstrated very high interrater reliability (0.99), retest reliability (0.98-0.99), and internal consistency $(0.97)$ in patients with acquired brain injury, including children with tumors [58, 59]. Furthermore, the HiMAT did not exhibit a ceiling effect in children with an acquired brain injury [59].

\section{Functional muscle strength}

Functional muscle strength will be measured by identifying $30 \mathrm{~s}$ repetition maximum, preceded by a practice trial, for the following exercises: sit-to-stand, and halfkneel to stand, based on the protocol outlined in Verschuren et al. [60]. These assessments have evidence of reliability (ICC range: $0.84-0.98$ ) in children with acquired brain injuries (including brain tumors) [59].

\section{Habitual physical activity}

Daily moderate-to-vigorous physical activity will be measured using the ActiGraph GT3X+ triaxial accelerometer (ActiGraph Corporation, Pensacola, FL, USA). Monitors will be worn $24 \mathrm{~h}$ per day on their non-dominant wrist for seven consecutive days. Upon return of the accelerometer, the raw accelerometer data will be downloaded and processed into physical activity metrics using a random forest physical activity classification algorithm for children $[61,62]$. This validated algorithm uses patterns or features from the raw tri-axial acceleration signal to quantify daily time spent in sedentary activities (sitting or lying down), light-intensity activities and games, walking, running, and moderate-to-vigorous intensity activities and games. Non-wear time will be quantified by summing the prediction windows in which the standard deviation of the acceleration signal was $<13 \mathrm{mg}$ for $\geq 30$ consecutive minutes [63].

\section{Gait}

The GAITRite system (GAITRite Electronic Walkway, CIR Systems Inc.) will be used to measure real-time temporal and spatial parameters of gait, including velocity, cadence, step length, stride length, heel-to-heel base of support, single leg support (left and right), double leg support, and toe in/out angles [64]. Participants will walk at a self-selected comfortable walking speed across a pressure-sensor mat. The GAITRite system is a valid and reliable tool for measuring both averaged and individual step parameters of gait in children, with ICC ranging between 0.57 and 0.93 [64, 65].

\section{Balance}

Balance will be assessed using a PASCO force plate (PASCO PASport 22-axis force platform PS-2142). Participants will be assessed in three stances; bilateral, right unilateral and left unilateral, under two conditions; barefoot contact and a foam surface placed on the force plate. Displacement of center of pressure and mean sway velocity in mediolateral and anteroposterior directions will be measured $[66,67]$.

\section{Peripheral neuropathy}

A modified battery of the pediatric-modified Total Neuropathy Scale (peds-mTNS) will assess lower extremity neurological signs and symptoms, to evaluate their contribution to movement and balance findings. The pedsmTNS comprises three sets of questions on sensory symptoms, pain, motor function and autonomic function, as well as a five-part non-invasive neurological exam, including light touch sensation, vibration sensation, pin sensation, distal muscle strength and deep tendon reflexes [68]. The ped-mTNS demonstrated excellent internal consistency $(>0.9)$ in school-aged children with chemotherapy-induced peripheral neuropathy [68]. To minimize potential confounding factors of central nervous system signs, plantar (Babinski) and clonus reflexes will also be tested $[69,70]$.

\section{Quality of life (QoL)}

The Pediatric Quality of Life Inventory (PedsQL) Generic Core Scales 4.0 comprise 23 items measuring four dimensions of QoL; physical functioning, emotional functioning, social functioning and school functioning [71]. Each item utilizes a 5-point Likert response scale across child self-report and parent proxy-report $(0=$ never a problem to; $4=$ almost always a problem), which will be reversed scored, with higher scores indicating better QoL. The measure consists of three age groups: 5 to 7 years (assessor-administered), eight to 12 years, and 
13 to 17 years. The PedsQL will be completed by both parents and participants, with both child- and parentreport total scale scores reporting internal consistency of 0.90 [71]. This measure has demonstrated reliability and validity in samples of healthy children and pediatric patients with acute or chronic health conditions (including cancer) [72-74].

Parents and children will also complete the PedsQL Brain Tumor Module [74]. This disease-specific module includes 24 items encompassing six subscales; cognitive problems, pain and hurt, movement and balance, procedural anxiety, nausea and worry. To ensure all questions are considered age-appropriate, the child-report measure excludes the item "trouble writing school papers and reports", for ages five to seven. The PedsQL Brain Tumor Module has demonstrated reliability and validity in a sample of children with brain tumors, with internal consistency of $0.78-0.92$ for the parent-report score and $0.76-0.87$ for child self-report score [73].

\section{Fatigue}

Pediatric Quality of Life Inventory Multidimensional Fatigue Scale (PedsQL-MFS) will be completed by parents and children, including 18 items encompassing three subscales; general fatigue, sleep/rest fatigue, and cognitive fatigue. The format, instructions and Likert response scale are identical to the PedsQL Generic Core Scales, with higher scores indicating less fatigue. PedsQL-MFS has demonstrated internal consistency, reliability and validity among a diverse sample of pediatric cancer patients (aged 2-18) [74]. Validity has been demonstrated in comparisons with healthy, non-cancer pediatric populations [74].

\section{Participation}

The Participation and Environment Measure for Children and Youth (PEM-CY) is a 20-item instrument completed by the parent and examines the frequency and level of participation across three settings; home, school and community [75]. For each item, parents will be asked to rate: (1) the frequency of their child's participation in the stated activity on an 8-point scale (daily to never); (2) how involved their child typically is while participating on a 5-point scale (very involved to minimally involved); and (3) whether the parent would like to see a change in their child's level of participation (yes/no). If yes, the parent will be asked what kind of change they would like to see on a 5-point scale (more involved to less involved). After each section, parents will be asked to indicate whether certain features of the environment make it easier or harder for their child to participate. This measure has demonstrated reliability and validity in a sample of children and youth with and without disabilities (aged 5-17 years), with acceptable internal consistency [75].

\section{Perceived movement competence}

The Perceived Movement Skill Competence for Young Children is a pictorial tool that scores a child's perceived movement competence for fundamental movement skills [76]. It involves the participant looking at picture cards of fundamental movement skills that include six locomotor items (run, gallop, hop, leap, jump, glide) and six object control items (strike a ball, bounce a ball, catch, kick, overarm throw and underarm roll). Each card shows a picture of a 'poor' or 'good' performance of that skill and the participant will be asked to choose which picture they most identify with. In a sample of 23 children, internal consistency was acceptable $(\geq 0.60)$ for both locomotor and object control [76]. Test-re-test reliability over a one-week period was excellent $(\geq 0.83)$ [76].

\section{Parameters of body composition}

Total body scans using dual-energy X-ray absorptiometry (DXA; Prodigy, GE Medical Systems, LUNAR, Madison, WI, USA) will acquire parameters of bone mineral density (BMD, $\mathrm{g} / \mathrm{cm}^{2}$ ) and body composition (total body lean and fat mass, $\mathrm{kg}$ ) data. Scans of the anterior-posterior spines L1-L4, left and right proximal femur will additionally be acquired. All scans will be performed as per standard protocols in lying supine and analyzed using the manufacturers pediatric software (EnCore version 14.1). Total body lean mass will be calculated as fat-free mass minus bone mineral content. DXA measured parameters of body composition and bone mineral density demonstrate a coefficient of variation of less than $2 \%[77,78]$.

\section{Sample size and power}

To detect the clinically important differences of $5.5 \mathrm{~mL} /$ $\mathrm{kg} / \mathrm{min}$ for Peak $V_{2}$ [79] and 2 points on the COPM [48] with $80 \%$ power, a sample of 20 participants per group is required $(n=40)$. This calculation assumes a two-tailed alpha level of 0.05 , and standard deviations of $5.5 \mathrm{~mL} / \mathrm{kg} / \mathrm{min}$ and 1.5 points for peak $V \mathrm{O}_{2}$ [80] and the COPM score [81], respectively. To offset a projected attrition rate of up to $20 \%$, we will recruit and enroll 24 participants per group, providing an overall sample size of 48 .

\section{Statistical analysis}

Analysis will follow standard principles for randomized controlled trials using two-group comparisons including all participants on an intention-to-treat basis. Betweengroup differences on the primary and secondary outcomes will be tested immediately post-intervention and at 6- and 12-month follow-up assessments using linear 
regression, with treatment group (exercise vs. usual care control) as the main effect and baseline measures of the respective outcome, along with other potential confounders (e.g., age, sex), as covariates.

\section{Ethics}

Ethical approval has been granted from the human research ethics committee of Children's Health Queensland (HREC/19/QCHQ/50464) and the Queensland University of Technology (1900000696). The trial has been prospectively registered with the Australian New Zealand Clinical Trials Registry (ACTRN12619000841178). Before enrolling in the trial, full written and informed consent will be obtained from legal parents/guardians of all participants, verbal assent for children aged seven to 13 years, and verbal and written assent for children aged 14 years or older. Participant data will be managed on a secured electronic database (REDCap) and hardcopy forms stored securely at the research facility.

\section{Safety and adverse events}

A standardized protocol will be used to monitor adverse events that may occur during the face-to-face exercise sessions or home-based program. Adverse events commonly related to participating in physical activity may include, but are not limited to muscle soreness, falls, injury, exacerbation of existing symptoms, or any new or changed health problems since the previous contact. Adverse events will be graded using the Common Terminology Criteria for Adverse Events version 5.0 criteria [82]. Serious adverse events related to the intervention will be reported by the chief investigator to the lead ethics committee. Risk assessments with mitigation strategies will be implemented prior to commencing the home-based program.

\section{Discussion}

This paper presents the rationale and design for a randomized controlled trial evaluating the efficacy of a 12week goal-directed therapeutic exercise intervention in pediatric survivors of PFBTs. The increasing incidence and survival rates of PFBT indicate that there is a growing population of paediatric survivors of PFBT. However, there is a lack of research informing health care providers on effective interventions to manage the adverse effects of the disease and its treatment. To date, exercise interventions in childhood cancer survivors have implemented a structured exercise training approach, in group- and/or home-based settings. No studies have evaluated the efficacy of a goal-directed exercise intervention in any paediatric brain tumour survivor cohort, which may be associated with larger, more sustainable improvements physical functioning and habitual physical activity. Improvements in cardiorespiratory fitness and goal attainment may enhance the quality of survivorship and play a major role in mitigating the risk of disabling secondary health conditions later in life such as obesity, cardiovascular disease, osteoporosis, and metabolic disorders. The program of research will have strong potential for translation to clinical practice and pediatric rehabilitation services.

\section{Abbreviations \\ BMD: Bone mineral density; COPM: Canadian Occupational Performance Measure; CTCAE: Common terminology criteria for adverse events; DXA: Dual-energy X-ray absorptiometry; HiMAT: High-level mobility assessment tool; ICF: International Classification for Functioning, Disability and Health; PACTS: Physical activity in survivorship; Ped-mTNS: Pediatric- Modified Total Neuropathy Scale; Peds-QL: Pediatric Quality of Life Inventory; Peds-QL MFS: Pediatric quality of life inventory multidimensional fatigue scale; PEM-CY: Participation and environment measure for children and youth; PFBT: Posterior fossa brain tumor; PMSC: Perceived movement competence skill scale; QoL: Quality of Life; QUT: Queensland University of Technology; $\mathrm{VO}_{2}$ : Volume of oxygen consumed in one minute; $\mathrm{VCO}_{2}$ : Volume of carbon dioxide expired in one minute; SPIRIT: Standard Protocol Items: Recommendations for Interventional Trials}

\section{Supplementary Information}

The online version contains supplementary material available at https://doi. org/10.1186/s12887-021-02566-7.

Additional file 1. Standard Protocol Items: Recommendations for Intervention Trials (SPIRIT) Checklist". The document contains a table listing the SPIRIT checklist criteria and the page number/s where each criterion is addressed in the manuscript.

\section{Acknowledgements \\ The authors would like to thank the Children's Brain Cancer Centre, particularly Professor Greig de Zubricaray (QUT), for their on-going support in this study. In addition, the authors would like to acknowledge the contribu- tions of the Clinical Nurse Consultants at the Children's Health Queensland Hospital and Health Service for their assistance with recruitment, and Amanda James, Clinical Research Manager at the Centre for Children's Health Research, for assistance with on-site facility coordination.}

\section{Authors' contributions}

SGT, EB, CXS, and TH were responsible for the original conceptual design of the study. DSKB, CT and MR contributed to measurement protocols and study logistics. BEK drafted the manuscript and was critically reviewed by authors SGT, EB and CXS. All authors read and approved the final manuscript.

\section{Funding}

The Physical ACTivity in Survivorship (PACTS) study is supported by a project grant from the Children's Brain Cancer Centre through the Children's

Hospital Foundation (CCABCR010). The funders had no role in the design of the study or preparation of the manuscript, and will remain separate for data collection, analysis, and interpretation of the data.

\section{Availability of data and materials}

Not applicable.

\section{Declarations}

Ethics approval and consent to participate

Ethical approval has been granted from the human research ethics committees of Children's Health Queensland (HREC/19/QCHQ/50464) and QUT (1900000696). Full written and informed consent obtained from legal parents/guardians of all participants. 


\section{Consent for publication}

Not applicable.

\section{Competing interests}

The authors declare no competing interests.

\section{Author details}

'Institute of Health and Biomedical Innovation at the Queensland Centre for Children's Health Research, Queensland University of Technology (QUT), Brisbane, Queensland, Australia. ${ }^{2}$ School of Exercise and Nutrition Science, Queensland University of Technology (QUT), Brisbane, Queensland, Australia. ${ }^{3}$ School of Allied Health Sciences, Griffith University, Gold Coast, Queensland, Australia. ${ }^{4}$ UNSW Fatigue Research Program, Kirby Institute, University of New South Wales, Sydney, NSW, Australia. ${ }^{5}$ School of Clinical Sciences,

Queensland University of Technology (QUT), Brisbane, Queensland, Australia.

${ }^{6}$ Queensland Children's Hospital, Brisbane, Queensland, Australia.

\section{Received: 18 January 2021 Accepted: 22 February 2021} Published online: 01 March 2021

\section{References}

1. Poussaint TY. Chapter 46 - Pediatric brain Tumors. In: Newton HB, Jolesz FA, editors. Handbook of Neuro-oncology Neurolmaging. San Diego: Academic Press; 2008. p. 469-84.

2. Smith MA, Seibel NL, Altekruse SF, Ries LA, Melbert DL, O'Leary M, et al. Outcomes for children and adolescents with Cancer. J Clin Oncol. 2010; 28(15):2625-34.

3. Brandão LA, Young PT. Posterior Fossa Tumors. Neuroimaging Clin. 2017; 27(1):1-37.

4. Youlden D, Aitken J. Childhood cancer in Australia 1983-2015. Brisbane: Cancer Council Australia; 2019

5. Hanzlik E, Woodrome SE, Abdel-Baki M, Geller TJ, Elbabaa SK. A systematic review of neuropsychological outcomes following posterior fossa tumor surgery in children. Childs Nerv Syst. 2015;31(10):1869-75.

6. Long TM, Rath SR, Maroni TD, Wallman KE, Atkinson HC, Gottardo NG, Cole $\mathrm{CH}$, Choong CS, Naylor LH. Fitness, body composition and vascular health in adolescent and young adult survivors of paediatric brain cancer and cranial radiotherapy. Int J Adolesc Med Health. 2017:31(5):/j/ijamh.2019.31.issue-5/ ijamh-2017-0082/ijamh-2017-0082.xml. https://doi.org/10.1515/ijamh-20170082. PMID: 28930627.

7. Odame I, Duckworth J, Talsma D, Beaumont L, Furlong W, Webber C, et al. Osteopenia, physical activity and health-related quality of life in survivors of brain tumors treated in childhood. Bone. 2005;36:S43-S.

8. Piscione PJ, Bouffet E, Mabbott DJ, Shams I, Kulkarni AV. Physical functioning in pediatric survivors of childhood posterior fossa brain tumors. Neuro-Oncol. 2014;16(1):147-55.

9. Lassaletta A, Bouffet E, Mabbott D, Kulkarni AV. Functional and neuropsychological late outcomes in posterior fossa tumors in children. Childs Nerv Syst. 2015;31(10):1877-90.

10. Macartney G, Stacey D, Harrison MB, VanDenKerkhof E. Symptoms, coping, and quality of life in pediatric brain tumor survivors: a qualitative study. Oncol Nurs Forum. 2014;41(4):390-8.

11. Schulte F, Russell KB, Cullen P, Embry L, Fay-Mcclymont T, Johnston D, et al. Systematic review and meta-analysis of health-related quality of life in pediatric CNS tumor survivors. Pediatr Blood Cancer. 2017:64(8):n/a-n/a.

12. Bell H, Ownsworth $T$, Owen $L$, Sheeran N, Chambers S. A systematic review of factors related to children's quality of life and mental health after brain tumor. Psycho-Oncol. 2018;27(10):2317-26.

13. Gunn ME, Mört S, Arola M, Taskinen M, Riikonen P, Möttönen M, et al. Quality of life and late-effects among childhood brain tumor survivors: a mixed method analysis. Psycho-Oncol. 2016;25(6):677-83.

14. Ho KY, Li WHC, Lam KWK, Wei X, Chiu SY, Chan C-FG, et al. Relationships among fatigue, physical activity, depressive symptoms, and quality of life in Chinese children and adolescents surviving cancer. Eur J Oncol Nurs. 2019. 38:21-7.

15. Van Dijk-Lokkart EM, Steur LMH, Braam KI, Veening MA, Huisman J, Takken T, et al. Longitudinal development of cancer-related fatigue and physical activity in childhood cancer patients. Pediatr Blood Cancer. 2019:66(12): e27949
16. Dengel D, Kelly A, Zhang L, Hodges J, Baker KS, Steinberger J. Signs of early sub-clinical atherosclerosis in childhood Cancer survivors. Pediatr Blood Cancer. 2014;61(3):532-7.

17. Gunn ME, Lähdesmäki T, Malila N, Arola M, Grönroos M, Matomäki J, et al. Late morbidity in long-term survivors of childhood brain tumors: a nationwide registry-based study in Finland. Neuro-Oncol. 2015;17(5):747-56.

18. Bereket A. Endocrinologic consequences of Pediatric posterior Fossa tumours. J Clin Res Pediatr Endocrinol. 2015;7(4):253-9.

19. Meacham LR, Chow EJ, Ness KK, Kamdar KY, Chen Y, Yasui Y, et al. Cardiovascular risk factors in adult survivors of pediatric cancer--a report from the childhood cancer survivor study. Cancer Epidemiol Biomark Prev. 2010;19(1):170-81

20. Wolfe RK, Hunter RG, Madan-Swain TA, Reddy KA, Baños KJ, Kana KR. Cardiorespiratory fitness in survivors of Pediatric posterior Fossa tumor. J Pediatr Hematol Oncol. 2012;34(6):e222-e7.

21. Hoffman MC, Mulrooney DA, Steinberger J, Lee J, Baker KS, Ness KK. Deficits in physical function among Young childhood Cancer survivors. J Clin Oncol. 2013;31(22):2799-805.

22. Söntgerath R, Eckert K. Impairments of lower extremity muscle strength and balance in childhood Cancer patients and survivors: a systematic review. Pediatr Hematol Oncol. 2015:32(8):585-612.

23. Ness KK, Morris EB, Nolan VG, Howell CR, Gilchrist LS, Stovall M, et al. Physical performance limitations among adult survivors of childhood brain tumors. Cancer. 2010;116(12):3034-44.

24. Ness KK, Leisenring WM, Huang S, Hudson MM, Gurney JG, Whelan K, et al. Predictors of inactive lifestyle among adult survivors of childhood cancer. Cancer. 2009;115(9):1984-94.

25. Valentino K. Physical activity in survivors of Pediatric brain Tumors: a review. Pediatr Nurs. 2018:44(2):76-80.

26. Antwi G, Jayawardene W, Lohrmann D, Mueller E. Physical activity and fitness among pediatric cancer survivors: a meta-analysis of observational studies. Support Care Cancer. 2019;27(9):3183-94.

27. Remington P, Borwnson R. M. W. chronic disease epidemiology, prevention, and control. 4th edition. Ed. Remington PL, Brownson RC, Wegner MV, editors. Washington, DC: Alpha Press, an imprint of American Public Health Association; 2016

28. Braam Kl, van der Torre P, Takken T, Veening MA, van Dulmen-den Broeder E, Kaspers GJ. Physical exercise training interventions for children and young adults during and after treatment for childhood cancer. Cochrane Database Syst Rev. 2016:3:Cd008796.

29. Long TM, Rath SR, Wallman KE, Howie EK, Straker LM, Bullock A, et al. Exercise training improves vascular function and secondary health measures in survivors of pediatric oncology related cerebral insult. Plos One. 2018; 13(8):e0201449.

30. Rath SR, Long TM, Bear NL, Miles GCP, Bullock AM, Gottardo NG, et al. Metabolic and psychological impact of a pragmatic exercise intervention program in adolescent and Young adult survivors of Pediatric Cancerrelated cerebral insult. J Adolesc Young Adult Oncol. 2018;7(3):349-57.

31. Piscione PJ, Bouffet E, Timmons B, Courneya KS, Tetzlaff D, Schneiderman JE, et al. Exercise training improves physical function and fitness in long-term paediatric brain tumour survivors treated with cranial irradiation. Eur J Cancer. 2017:80:63-72

32. Ovans J, Hooke MC, Bendel AE, Tanner L. Physical therapist coaching to improve physical activity in children with brain Tumors: a pilot study. Pediatr Phys Ther. 2018;30(4):310-7.

33. Szulc-Lerch KU, Timmons BW, Bouffet E, Laughlin S, de Medeiros CB, Skocic $J$, et al. Repairing the brain with physical exercise: cortical thickness and brain volume increases in long-term pediatric brain tumor survivors in response to a structured exercise intervention. Neuroimage-Clin. 2018;18: 972-85.

34. Riggs L, Piscione J, Laughlin S, Cunningham T, Timmons BW, Courneya KS, et al. Exercise training for neural recovery in a restricted sample of pediatric brain tumor survivors: a controlled clinical trial with crossover of training versus no training. Neuro-Oncol. 2017;19(3):440-50.

35. Lam K, Li W, Chung O, Ho K, Chiu S, Lam H, et al. An integrated experiential training programme with coaching to promote physical activity, and reduce fatigue among children with cancer: a randomised controlled trial. Patient Educ Couns. 2018:101(11):1947-56

36. Li W, Ho K, Lam K, Lam H, Chui S, Chan G, et al. Adventure-based training to promote physical activity and reduce fatigue among childhood cancer survivors: a randomized controlled trial. Int J Nurs Stud. 2018;83:65-74. 
37. Löwing K, Bexelius A, Brogren CE. Activity focused and goal directed therapy for children with cerebral palsy - do goals make a difference? Disabil Rehabil. 2009;31(22):1808-16.

38. Jackman M, Lannin N, Galea C, Sakzewski L, Miller L, Novak I. What is the threshold dose of upper limb training for children with cerebral palsy to improve function? A systematic review. Aust Occup Ther J. 2020;67(3):26980.

39. Novak I, Mclntyre S, Morgan C, Campbell L, Dark L, Morton N, et al. A systematic review of interventions for children with cerebral palsy: state of the evidence. J Dev Med Child Neurol. 2013;55:885-910.

40. Willis C, Nyquist A, Jahnsen R, Elliott C, Ullenhag A. Enabling physical activity participation for children and youth with disabilities following a goaldirected, family-centred intervention. Res Dev Disabil. 2018;77:30-9.

41. Reedman S, Boyd RN, Sakzewski L. The efficacy of interventions to increase physical activity participation of children with cerebral palsy: a systematic review and meta-analysis. Dev Med Child Neur. 2017;59(10):1011-8.

42. Chan AW, Tetzlaff JM, Altman DG, Laupacis A, Gotzsche PC, Krle AJK, et al, SPIRIT 2013 statement: defining standard protocol items for clinical trials. Revista panamericana de salud publica $=$. Pan Am J Public Health. 2015; 38(6):506-14.

43. Palisano RJ, Chiarello LA, King GA, Novak I, Stoner T, Fiss A. Participationbased therapy for children with physical disabilities. Disabil Rehabil. 2012; 34(12):1041-52.

44. Organization WH. Global recommendations on physical activity for health. Geneva: World Health Organization; 2010.

45. Barker AR, Williams CA, Jones AM, Armstrong N. Establishing maximal oxygen uptake in young people during a ramp cycle test to exhaustion. $\mathrm{Br}$ J Sports Med. 2011;45(6):498-503.

46. Day JR, Rossiter HB, Coats EM, Skasick A, Whipp BJ. The maximally attainable VO2 during exercise in humans: the peak vs. maximum issue. J Appl Physiol. 2003;95(5):1901-7.

47. Welsman J, Bywater K, Farr C, Welford D, Armstrong N. Reliability of peak $\mathrm{VO}(2)$ and maximal cardiac output assessed using thoracic bioimpedance in children. Eur J Appl Physiol. 2005;94(3):228-34.

48. Law M, Polatajko H, Pollock N, McColl MA, Carswell A, Baptiste S. Pilot testing of the Canadian occupational performance measure: clinical and measurement issues. Can J Occup Ther. 1994;61(4):191-7.

49. Law MC, Baptiste S, Carswell A, McColl MA, Polatajko H, Pollock N. Canadian occupational performance measure: COPM: CAOT Publ. Ottawa: ACE; 1998.

50. Sakzewski L, Bleyenheuft Y, Boyd RN, Novak I, Elliott C, Reedman S, Morgan C, Pannek K, Fripp J, Golland P, Rowell D, Chatfield M, Ware RS. Protocol for a multisite randomised trial of Hand-Arm Bimanual Intensive Training Including Lower Extremity training for children with bilateral cerebral palsy: HABIT-ILE Australia. BMJ Open. 2019;9(9):e032194. https://doi.org/10.1136/ bmjopen-2019-032194. PMID: 31501133; PMCID: PMC6738737.

51. Cusick A, Lannin NA, Lowe K. Adapting the Canadian occupational performance measure for use in a paediatric clinical trial. Disabil Rehabil. 2007;29(10):761-6.

52. Baque E, Barber L, Sakzewski L, Boyd RN. Randomized controlled trial of web-based multimodal therapy for children with acquired brain injury to improve gross motor capacity and performance. Clin Rehabil. 2017;31(6): 722-32.

53. Wallen M, Ziviani J, Naylor O, Evans R, Novak I, Herbert RD. Modified constraint-induced therapy for children with hemiplegic cerebral palsy: a randomized trial. Dev Med Child Neurol. 2011;53(12):1091-9.

54. Bradley J, Howard J, Wallace E, Elborn S. Reliability, repeatability, and sensitivity of the modified shuttle test in adult cystic fibrosis. Chest. 2000; 117(6):1666-71.

55. Selvadurai HC, Cooper PJ, Meyers N, Blimkie CJ, Smith L, Mellis CM, et al. Validation of shuttle tests in children with cystic fibrosis. Pediatr Pulmonol. 2003;35(2):133-8.

56. Robertson RJ, Goss FL, Boer NF, Peoples JA, Foreman AJ, Dabayebeh IM, et al. Children's OMNI scale of perceived exertion: mixed gender and race validation. Med Sci Sports Exerc. 2000;32(2):452.

57. Cox NS, Follett J, McKay KO. Modified shuttle test performance in hospitalized children and adolescents with cystic fibrosis. J Cystic Fibros. 2006;5(3):165-70.

58. Williams GP, Greenwood KM, Robertson VJ, Goldie PA, Morris ME. High-level mobility assessment tool (HiMAT): interrater reliability, retest reliability, and internal consistency. Phys Ther. 2006;86(3):395-400.
59. Baque E, Barber L, Sakzewski L, Boyd R. Test-re-test reproducibility of activity capacity measures for children with an acquired brain injury. Brain Inj. 2016; 30(9):1143-9.

60. Verschuren O, Ketelaar M, Takken T, Van Brussel M, Helders PJ, Gorter JW. Reliability of hand-held dynamometry and functional strength tests for the lower extremity in children with cerebral palsy. Disabil Rehabil. 2008;30(18): 1358-66.

61. Chowdhury KA, Tjondronegoro GD, Chandran GV, Trost SG. Ensemble methods for classification of physical activities from wrist Accelerometry. Med Sci Sports Exerc. 2017;49(9):1965-73.

62. Trost SG, Zheng Y, Wong W. Machine learning for activity recognition: hip versus wrist data. Physiol Meas. 2014;35(11):2183-9.

63. Ahmadi MN, Nathan N, Sutherland R, Wolfenden L, Trost SG. Non-wear or sleep? Evaluation of five non-wear detection algorithms for raw accelerometer data. J Sports Sci. 2020;38(4):399.

64. Thorpe DE, Dusing SC, Moore CG. Repeatability of Temporospatial gait measures in children using the GAITRite electronic walkway. Arch Phys Med Rehabil. 2005;86(12):2342-6.

65. Baker BJ, Blount KD, Cox DE, Roberts BL. Reliability and validity of the GAIT Rite system for gait analysis in typically developing children. Pediatr Phys Ther. 2001;13(4):195.

66. Golriz S, Hebert JJ, Foreman KB, Walker BF. The reliability of a portable clinical force plate used for the assessment of static postural control: repeated measures reliability study. Chiropr Man Ther. 2012;20(1):14.

67. Lazzari RD, Politti F, Belina SF, Collange Grecco LA, Santos CA, Dumont AJL, et al. Effect of Transcranial direct current stimulation combined with virtual reality training on balance in children with cerebral palsy: a randomized, controlled, double-blind. Clin Trial J Motor Behav. 2017;49(3):329-36.

68. Gilchrist L, Tanner $L$. The pediatric-modified total neuropathy score: a reliable and valid measure of chemotherapy-induced peripheral neuropathy in children with non-CNS cancers. Support Care Cancer. 2013;21(3):847-56.

69. Dimitrijevic MR, Nathan PW, Sherwood AM. Clonus: the role of central mechanisms. J Neurol Neurosurg Psychiatry. 1980;43(4):321-32.

70. Walker HK. The Plantar Reflex. In: Walker HK, Hall WD, Hurst JW, editors. Clinical methods: the history, physical, and laboratory examinations. Boston: Butterworth Publishers; 1990.

71. Varni JW, Seid M, Kurtin PS. PedsQL 4.0: reliability and validity of the Pediatric quality of life inventory version 4.0 generic core scales in healthy and patient populations. Med Care. 2001;39(8):800-12.

72. Joschtel B, Gomersall S, Trost S. Fundamental movement skills among children with non-CF bronchiectasis. J Sci Med Sport. 2017;20:e63.

73. Palmer SN, Meeske KA, Katz ER, Burwinkle TM, Varni JW. The PedsQL brain tumor module: initial reliability and validity. Pediatr Blood Cancer. 2007; 49(3):287-93.

74. Varni JW, Burwinkle TM, Katz ER, Meeske K, Dickinson P. The PedsQL in pediatric cancer: reliability and validity of the Pediatric quality of life inventory generic Core scales, multidimensional fatigue scale, and Cancer module. Cancer. 2002;94(7):2090-106.

75. Coster W, Bedell G, Law M, Khetani MA, Teplicky R, Liljenquist K, et al. Psychometric evaluation of the participation and environment measure for children and youth. Dev Med Child Neurol. 2011;53(11):1030-7.

76. Barnett LM, Ridgers ND, Zask A, Salmon J. Face validity and reliability of a pictorial instrument for assessing fundamental movement skill perceived competence in young children. J Sci Med Sport. 2015;18(1):98-102.

77. Ellis K, Shypailo R, Pratt J, Mersmann H, Pond W. Accuracy of Dual-Energy XRay Absorptionmetry (DXA) for Body-Composition Measurements in Pediatric Studies. Faseb J. 1993;7(3):A359-A.

78. Laskey MA. Dual-energy X-ray absorptiometry and body composition. Nutrition. 1996;12(1):45-51. https://doi.org/10.1016/0899-9007(95)00017-8. PMID: 8838836.

79. Armstrong N, Welsman J. Development of peak oxygen uptake from 11-16 years determined using both treadmill and cycle ergometry. Eur J Appl Physiol. 2019;119(3):801-12.

80. Dubnov-Raz G, Azar M, Reuveny R, Katz U, Weintraub M, Constantini NW. Changes in fitness are associated with changes in body composition and bone health in children after cancer. Acta Paediatr. 2015;104(10):1055-61.

81. Law M, Anaby D, Imms C, Teplicky R, Turner L. Improving the participation of youth with physical disabilities in community activities: an interrupted time series design. Aust Occup Ther J. 2015;62(2):105-15. 
82. National Institutes of Health and National Cancer Institute. Common Terminology Criteria for Adverse Events (CTCAE). Version 5. U.S. Department of Health and Human Services; 2017. https://ctep.cancer.gov/ protocoldevelopment/electronic_applications/docs/CTCAE_v5_Quick_ Reference_8.5x11.pdf. Accessed 5 Aug 2020.

\section{Publisher's Note}

Springer Nature remains neutral with regard to jurisdictional claims in published maps and institutional affiliations.

- fast, convenient online submission

- thorough peer review by experienced researchers in your field

- rapid publication on acceptance

- support for research data, including large and complex data types

- gold Open Access which fosters wider collaboration and increased citations

- maximum visibility for your research: over $100 \mathrm{M}$ website views per year

At BMC, research is always in progress. 